\title{
- Report Toward Inter-isolated Island Cooperation for the Drip, Ship, and Retrieve Method in the Sakishima Islands: A Case Report
}

Naoki Wakuta, ${ }^{1,2}$ Satoshi Yamamoto, ${ }^{1}$ Shinobu Adachi, ${ }^{3}$ and Eiji Motonaga ${ }^{4}$

Objective: Based on the findings of preferable outcomes from recanalization therapy in recent studies, regional partnerships for the endovascular treatment of acute ischemic stroke are being promoted. However, reports of interisland cooperation between remote islands located far from high-volume centers on the mainland are rare.

Case Presentation: A 63-year-old man experienced an acute ischemic stroke on a small, isolated island in Okinawa, Japan. He was transferred by helicopter to the primary emergency hospital on Ishigaki Island, which was the nearest island on which he could be administered recombinant tissue plasminogen activator (rtPA). After this, he was carried again by helicopter and ambulance to the primary stroke center on Miyako Island using the drip and ship method. Mechanical thrombectomy with a stent retriever achieved recanalization of the occluded major vessels and improved the neurological disturbance. The patient became neurologically independent and could be discharged only 11 days after onset.

Conclusion: Building a local area network that includes hospitals providing mechanical thrombectomy is a meaningful approach to treating acute ischemic stroke occurring on isolated islands. It is necessary to recognize the specific restrictions imposed by helicopter transportation and to make efforts to shorten the time required for key processes to provide faster treatment.

Keywords DSR (drip, ship, and retrieve), endovascular treatment, Sakishima Islands, acute ischemic stroke, medical care system

\section{Introduction}

Patients suffering from acute cerebral infarction in remote areas located far from stroke centers may not have the opportunity to receive recanalization therapy for major vessel occlusion because of the restricted time window

${ }^{1}$ Department of Neurosurgery, Okinawa Miyako Hospital, Miyakojima, Okinawa, Japan

${ }^{2}$ Department of Neurosurgery, Fukuoka University Hospital; School of Medicine, Fukuoka University, Fukuoka, Fukuoka, Japan ${ }^{3}$ Department of Neurosurgery, Okinawa Yaeyama Hospital, Ishigaki, Okinawa, Japan

${ }^{4}$ Department of Rehabilitation, Internal Medicine, and Family Medicine, Okinawa Miyako Hospital, Miyakojima, Okinawa, Japan

Received: February 4, 2020; Accepted: March 13, 2020 Corresponding author: Naoki Wakuta. Department of Neurosurgery, Okinawa Miyako Hospital, 427-1 Shimozato, Hirara, Miyakojima, Okinawa 906-0013, Japan

Email:nwakuta@fukuoka-u.ac.jp

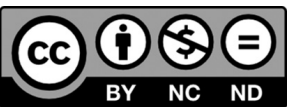

This work is licensed under a Creative Commons Attribution-NonCommercialNoDerivatives International License.

(C)2020 The Japanese Society for Neuroendovascular Therapy during which the required treatment has to occur. Thus, the challenge of providing suitable transportation for such patients continues in Japan, leading to the introduction of the drip, ship, and retrieve (DSR) method. ${ }^{1,2)}$ Recent clinical studies have reported good outcomes for acute cerebral infarction patients receiving mechanical thrombectomy, even more than 6 hours after onset, based on an appropriate indication. ${ }^{3,4)}$ On the basis of these findings, the guidelines for the early management of acute ischemic stroke patients by the American Heart Association were revised and the indication time for mechanical thrombectomy was extended in 2018. ${ }^{5)}$ Thus, it is anticipated that the likelihood of patients living on isolated islands receiving emergent endovascular therapy will increase, and that there will be more need to build regional partnerships. A previous report described a partnership established between a high-volume stroke center on the mainland and hospitals on remote islands in Japan ${ }^{1}$; however, thus far, reports of inter-isolated island cooperation for acute ischemic stroke have been rare. Here, we present the first case treated from the Sakishima Islands as part of an inter-island cooperation project. 


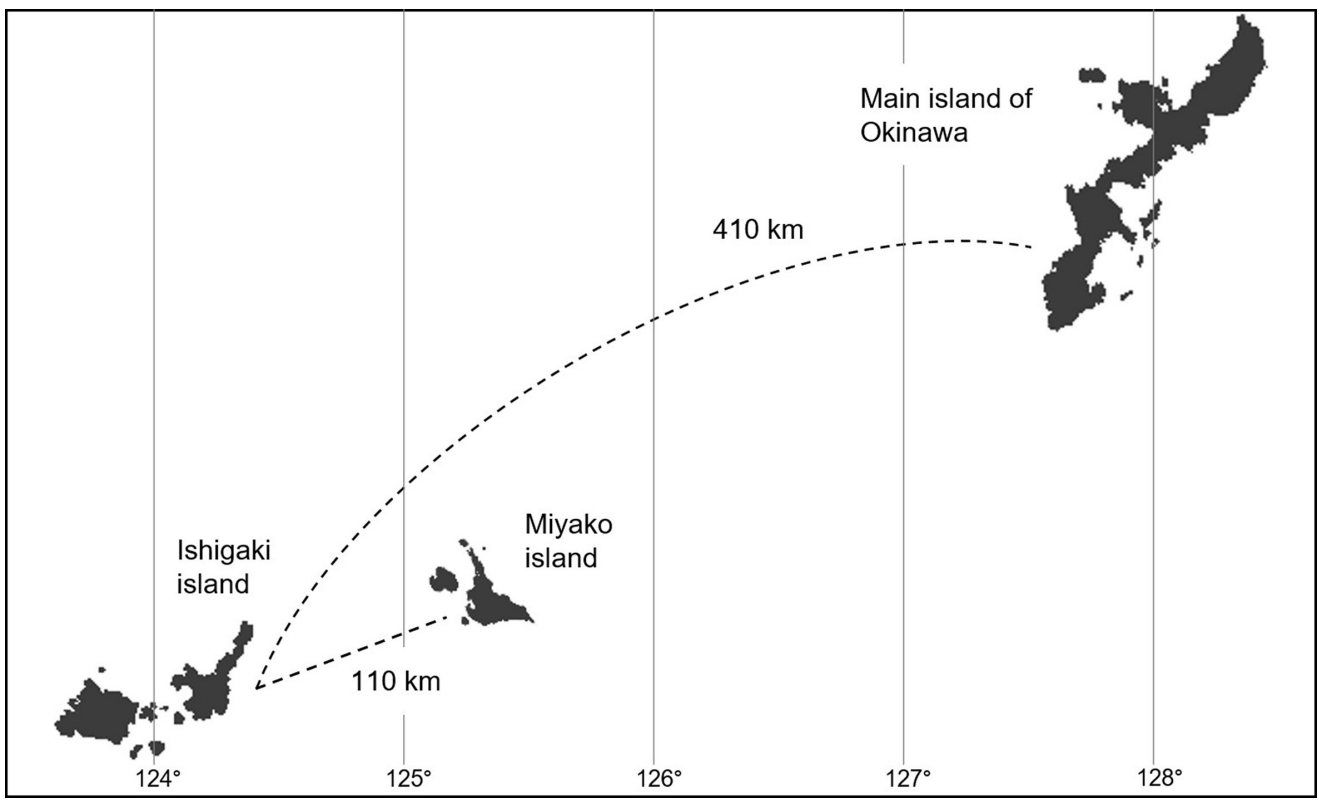

Fig. 1. Geographic location of the Sakishima Islands and the main island of Okinawa.

\section{Geographic and medical background of the Sakishima Islands}

Miyako Island is located at a latitude of $24^{\circ}$ north and a longitude of $125^{\circ}$ east, which is approximately $2,000 \mathrm{~km}$ southwest of Tokyo and $300 \mathrm{~km}$ from the main island of Okinawa. Ishigaki Island is located at a latitude of $24^{\circ}$ north and a longitude of $124^{\circ}$ east, which is $110 \mathrm{~km}$ west of Miyako Island (Fig. 1). Both Miyako and Ishigaki Islands are isolated islands belonging to the Sakishima Islands, along with several other remote, isolated islands, in Okinawa Prefecture, which is located in a distant region of southwest Japan. Both Okinawa Miyako Hospital on Miyako Island and the Okinawa Yaeyama Hospital on Ishigaki Island are only prefectural hospitals. In both hospitals, emergency medical treatment for cerebrovascular stroke is available; however, emergency neuroendovascular treatment can only be performed at Okinawa Miyako Hospital.

\section{Partnership between the Sakishima Islands}

While transportation from Ishigaki Island to the main island of Okinawa requires 2 hours, it only takes $40 \mathrm{~min}$ to Miyako Island. To improve the treatment of acute cerebral infarction, the Sakishima inter-island partnership agreement was reached in May 2019 through cooperation between the relevant administrative organizations. The aim of the partnership is to introduce the DSR method using helicopter transportation between the Sakishima Islands.

\section{Case Presentation}

A 63-year-old man presented with consciousness disturbance, aphasia, dysarthria, and right hemiparesis at 12:30 $\mathrm{pm}$ while on a small, isolated island with a population of 360 and only one clinic. He was carried by helicopter from the clinic to the Okinawa Yaeyama Hospital on Ishigaki Island at 2:13 pm, $103 \mathrm{~min}$ after onset. The patient scored 17 on the National Institute of Health Stroke Scale (NIHSS). His electrocardiogram showed atrial fibrillation, while computed tomography (CT) revealed hyperdense middle cerebral artery (MCA) signs and a light, low-density area in the left MCA territory; the Alberta Stroke Program Early CT (ASPECT) score for the patient was 7. Occlusion of the left MCA was observed on an additional enhanced CT. After administration of recombinant tissue plasminogen activator (rtPA) was begun at 3:30 pm (onset-to-needle time: $180 \mathrm{~min}$ ), the patient was carried by helicopter and ambulance to Okinawa Miyako Hospital, 333 min after onset (at 6:03 pm). His neurological manifestations had not improved, however, as the CT findings on admission were almost the same as those recorded at Okinawa Yaeyama Hospital. Thus, it was determined that endovascular surgery was indicated. Under local anesthesia, the right femoral artery was punctured with an $8 \mathrm{Fr}$ sheath (onset-topuncture time: $347 \mathrm{~min}$ ). A balloon guide catheter ( $8 \mathrm{Fr}$ Optimo; Tokai Medical Products, Aichi, Japan) was guided into the left internal carotid artery. Angiography showed occlusion of the left M1 segment of the MCA (Fig. 2). 

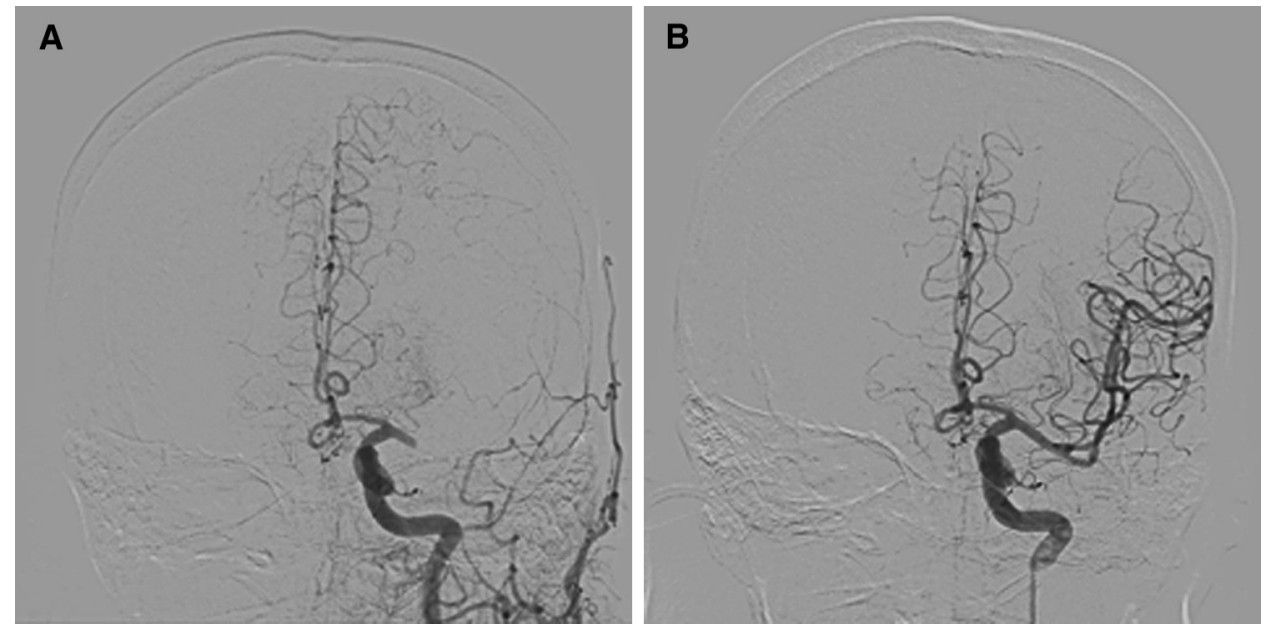

Fig. 2. (A) Cerebral angiography before treatment revealed occlusion of the left MCAM1 segment. (B) Complete recanalization was achieved after mechanical thrombectomy. MCA: middle cerebral artery

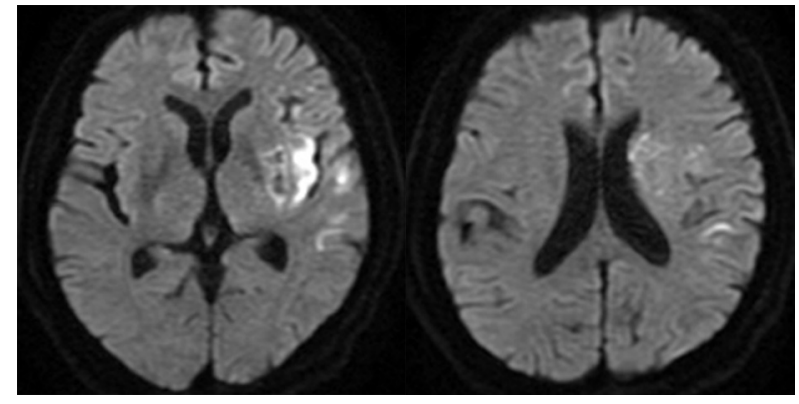

Fig. 3. Postoperative MRI using DWI. DWI: diffusion-weighted imaging; MRI: magnetic resonance imaging

A Marksman microcatheter (Medtronic, Minneapolis, MI, USA) was passed into the left M2 segment over a CHIKAI 14 microwire (Asahi Intec, Aichi, Japan), and a Solitaire Platinum $4 \mathrm{~mm} \times 40 \mathrm{~mm}$ revascularization device (Medtronic, Irvine, CA, USA) was deployed across the occlusion site. Immediate flow restoration of the MCA was achieved, with the partial thrombus attached to the removed stent retriever. Recanalization of the left M1 segment was observed by angiography, but the M2 segment remained occluded. The Marksman microcatheter and CHIKAI 14 microwire were delivered to the occlusion site again, and the Solitaire was deployed across the occlusion from the M2 to the M1 segment. With the successful removal of the thrombus followed by an immediate flow restoration, total recanalization at thrombolysis in cerebral infarction (TICI) grade 3 was achieved (puncture-to-recanalization time: 101 min) (Fig. 2). After mechanical thrombectomy, the neurological manifestations had improved to mild aphasia, dysarthria, and right hemiparesis (NIHSS 6). Postoperative MRI showed only partial brain infarctions in the left MCA territory, which were also detected on the preoperative CT (Fig. 3). On the next day of endovascular treatment, the neurological manifestations had recovered further: only mild aphasia and dysarthria remained (NIHSS 2). The patient was able to begin aggressive rehabilitation protocol and was independent in his daily activities postoperatively after several days. The patient was flown back to Okinawa Yaeyama Hospital by airplane to continue his rehabilitation at 11 days after onset.

\section{Discussion}

We found that the DSR method was useful in the present case, as has been reported in previous studies. ${ }^{1,6,7)}$ However, this study also revealed a number of problems of our inter-island partnership which require discussion.

\section{Helicopter transportation}

To introduce the DSR method between isolated islands, the only option for emergency transportation is the helicopter. The application of the DSR method in our inter-island partnership is especially unique because multiple helicopter flights are often required between the isolated islands, as was true for the case presented here. The Sakishima inter-island cooperation project used a Japan Coastguard (JCG) helicopter to transport the patient. The adaptability of this helicopter is useful because it is able to fly even at night or in bad weather. However, although the speed and safety of helicopter flight for the drip and ship method has been previously demonstrated, ${ }^{1,8)}$ one problem with the helicopter is the inevitably greater loss of time during the request to departure process for each flight, as compared 
with other transportation methods, such as ambulance. Furthermore, reassigning a helicopter that has arrived so that it can be used for transport onward to the next hospital, in the same way as is possible with an ambulance, is difficult. Additionally, the JCG helicopter base is not located on any of the other individual remote islands, but rather only on Ishigaki Island. Thus, for the transportation to Okinawa Yaeyama hospital, the time in which the JCG helicopter is in use often comprises one round trip between Ishigaki Island and the remote islands.

\section{Door-in-door-out time}

The 157 min door-in-door-out (DIDO) time for the present case is longer than the reported reference time of 45-75 $\mathrm{min}^{6,7)}$; however, the DIDO time is a key period during which it is important to save time in the DSR method. Previous studies have revealed that the period between the diagnosis and the request for transportation is what lasts the longest throughout the whole DIDO time, both in cases of ambulance and helicopter transportation. ${ }^{6,9)}$ In fact, the time between the CT and the request for transportation was $90 \mathrm{~min}$, which was the longest period making up the DIDO time of the present case. This included 50 min of actual decision-making regarding the acute endovascular treatment, in addition to a preventable 40-min loss of time, which was caused by a failure to follow the correct work flow in requesting the helicopter. Additionally, it is important to recognize that wasting more time on the request to departure process may extend the DIDO time required for helicopter transportation beyond that required for an ambulance. $\mathrm{Ng}$ et al. ${ }^{6}{ }^{6}$ reported the mean time of the retrieval request to departure as being $27 \mathrm{~min}$ for ambulance transportation; however, it was $47 \mathrm{~min}$ in the present case and in general, the preparation process for the JCG helicopter requires 40-60 min.

\section{Supportive tools and systems}

The lack of supportive tools and systems was another problem in our partnership. The introduction of common supportive tools or paradigms has also been reported to be a key aspect of the DSR method for island regions. ${ }^{1)}$ Teleradiology and telemedicine systems have recently focused on helping with diagnosis or with sharing patient information. ${ }^{1,5)}$ However, we have not yet managed to introduce common tools for sharing medical information in our cooperation project. Furthermore, while there is currently a paradigm for the treatment of acute ischemic stroke at Okinawa Miyako hospital, there is no common paradigm among the hospitals of the Sakishima Islands. A common paradigm would help not only to clarify the duty task, target time, and the process as a whole but also allow the education of medical staff in each hospital regarding the drip and ship method. ${ }^{10,11)}$ McTaggart et al. ${ }^{7)}$ reported that the design and sharing of a useful paradigm shortened the total time, including saving DIDO time, and improved the outcome. The design of a common paradigm will also help to reduce mistakes, such as those that occurred in our case.

\section{Conclusion}

The DSR method enabled by the inter-island cooperation was effective at treating a patient with severe acute cerebral infarction, achieving recanalization of their major vessels and a remarkable subsequent neurological improvement. The limitations of helicopter transportation and the multiple additional flights required may lead to a greater loss of time compared with other transportation methods. More effort is needed to shorten the time required for key processes and to introduce supportive tools and systems.

\section{Acknowledgment}

We thank Doran Amos, PhD, from Edanz Group (https:// en-author-services.edanzgroup.com/) for editing a draft of this manuscript.

\section{Disclosure Statement}

The first author and co-authors have no conflicts of interest.

\section{References}

1) Hiu T, Ozono K, Kawahara I, et al: Efficacy of the drip and ship method in 24-h helicopter transportation and teleradiology for isolated Islands. Neurol Med Chir (Tokyo) 2019; 59: 504-510.

2) Hiyama N, Yoshimura S, Shirakawa M, et al: Safety and effectiveness of drip, ship, and retrieve paradigm for acute ischemic stroke: a single center experience. Neurol Med Chir (Tokyo) 2016; 56: 731-736.

3) Nogueira RG, Jadhav AP, Haussen DC, et al: Thrombectomy 6 to 24 hours after stroke with a mismatch between deficit and infarct. N Engl J Med 2018; 378: 11-21.

4) Albers GW, Marks MP, Kemp S, et al: Thrombectomy for stroke at 6 to 16 hours with selection by perfusion imaging. N Engl J Med 2018; 378: 708-718.

5) Powers WJ, Rabinstein AA, Ackerson T, et al: Guidelines for the early management of patients with acute ischemic 
stroke: 2019 update to the 2018 guidelines for the early management of acute ischemic stroke: a guideline for healthcare professionals from the American Heart Association/American Stroke Association. Stroke 2019; 50: e344-e418.

6) $\mathrm{Ng}$ FC, Low E, Andrew E, et al: Deconstruction of interhospital transfer workflow in large vessel occlusion: real-world data in the thrombectomy era. Stroke 2017; 48: 1976-1979.

7) McTaggart RA, Yaghi S, Cutting SM, et al: Association of a primary stroke center protocol for suspected stroke by large-vessel occlusion with efficiency of care and patient outcomes. JAMA Neurol 2017; 74: 793-800.

8) Faine BA, Dayal S, Kumar R, et al: Helicopter "drip and ship" flights do not alter the pharmacological integrity of rtPA. J Stroke Cerebrovasc Dis 2018; 27: 2720-2724.

9) Schneider MA, McMullan JT, Lindsell CJ, et al: Reducing door-in door-out intervals in helicopter ST-segment elevation myocardial infarction interhospital transfers. Air Med J 2017; 36: 244-247.

10) Mansoor S, Zand R, Al-Wafai A, et al: Safety of a "drip and ship" intravenous thrombolysis protocol for patients with acute ischemic stroke. J Stroke Cerebrovasc Dis 2013; 22: 969-971.

11) Tekle WG, Chaudhry SA, Hassan AE, et al: Drip-and-ship thrombolytic treatment paradigm among acute ischemic stroke patients in the United States. Stroke 2012; 43: 1971-1974. 